\title{
Straight-Line Drawings of Protein Interactions System demonstration
}

\author{
Wojciech Basalaj ${ }^{1}$ and Karen Eilbeck ${ }^{2}$ \\ 1 University of Cambridge Computer Laboratory \\ Pembroke Street \\ Cambridge CB2 3QG, UK \\ wb204@cl.cam.ac.uk \\ 2 University of Manchester Biochemistry Division \\ 2.205 Stopford Building, Oxford Road \\ Manchester M13 9PT, UK \\ mobfeke1@fs2.scg.man.ac.uk
}

\begin{abstract}
This paper presents the first attempt at automatically generating drawings of protein interaction graphs. Such graphs are large and not necessarily connected. A straight-line drawing method based on the spring embedder metaphor has been found highly suitable for this task. The drawings produced exhibit edge length uniformity, even vertex distribution, and preserve graph topology well. This method is capable of generating both two- and three-dimensional layouts. A preliminary evaluation has been carried out based on a representative collection of interaction graphs.
\end{abstract}

\section{Introduction}

Protein-protein interaction data can be represented as a graph, with individual proteins taken as vertices, and the existence of an interaction between a pair of proteins denoted by an edge. These data are inherently complex, and graph drawing techniques can be used to convey the underlying structure effectively. Visual representation is an ideal method to communicate protein-protein interactions within the biological community.

This paper starts by giving the motivation for using visualisation techniques to analyse protein interaction data. Our approach is outlined and set in the context of related work. We then go on to discuss the details of the chosen graph drawing method, and show its suitability to the class of graphs typical of this domain. Our experiences with applying this method follow. We present insights contributed by domain experts when exploring protein interaction drawings. Finally, we speculate on future directions of our work, and offer some concluding remarks.

\section{Biological Context}

The genome projects such as Yeast [14], C. elegans [12], human [8], and E. coli [5], have produced a lot of biological data, pinpointing genes to places on the 
chromosomes, discovering analogies between species, locating disease genes. This data in itself is immense, but it is the post genome sequencing analysis that will produce vast amounts of complex experimental results. It is an attempt to make sense of the cells, tissues, organs and organisms that make use of the genetic load they are given. The analysis of protein-protein interactions endeavours to bring understanding to the function of both individual proteins and networks of proteins within the cellular context. An estimate is that about $40 \%$ of genes from sequencing projects are novel and have no assigned function [7]. Knowing the interactions that these proteins make can help to pinpoint the role that they play. The data is being gathered in both small scale experiments and large industrial size ventures, such as the plan to use the Yeast two hybrid method to map all of the interactions made by each of the 6000 proteins in yeast [13].

Currently the data from these experiments is reproduced mainly as tables of interactions, as displayed by the MIPS yeast genome resource centre [18]. Protein linkage maps have been produced for small genomes such as the bacteriophage T7 [2], but again this information is displayed in a tabular form. Specific databases such as Ecocyc, the E coli Metabolic Database, have represented pathways of interacting enzymes as automatically generated hierarchical diagrams [16].

The INTERACT database of protein-protein interactions [11] has been used as an information resource. The data held is of diverse interaction types: structural, enzymic, transient, permanent, therefore the corresponding graphs are undirected, unlike for the metabolic data. Two subsets of data have been used, Yeast, and E. coli, both fully sequenced unicellular organisms. The data from both of these subsets is of high cardinality and connectivity, so it is obvious that a pictorial representation via a straight-line drawing is best. Figures 3 and 4 are example visualisations of yeast interactions.

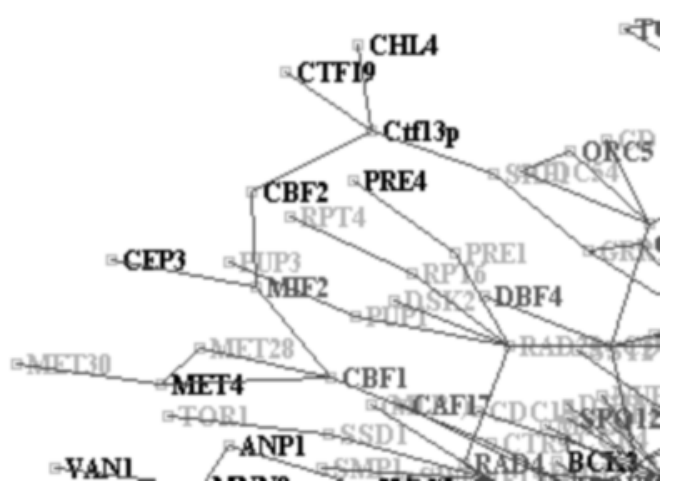

Fig. 1. Details of interactions in yeast with those proteins not present in C. elegans highlighted in black. The grey labelled proteins are only found once in C. elegans, whereas the light grey proteins have been replicated several times in the new genome

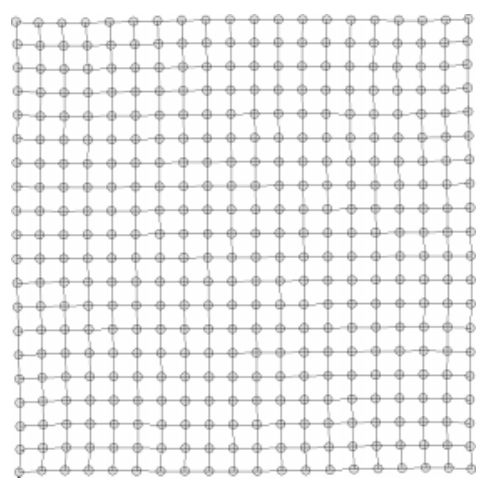

Fig. 2. 2D drawing of 20x20 grid 


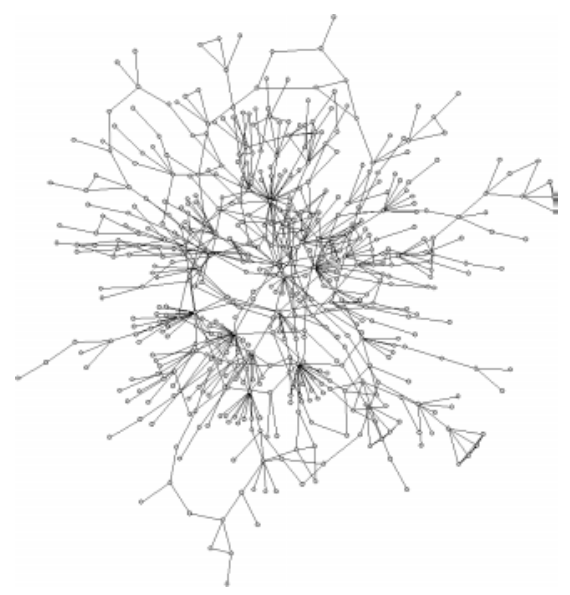

Fig. 3. Main component of interactions in yeast represented as a straight-line drawing in $2 \mathrm{D}$. There are 525 vertices and 724 edges in this graph

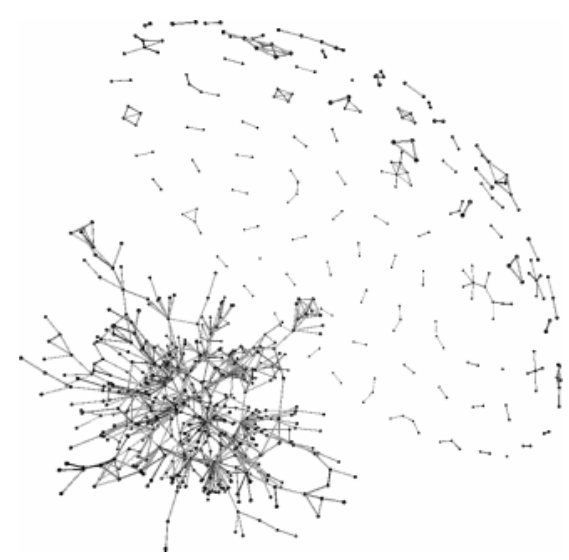

Fig. 4. 3D representation of interactions in yeast. There are 766 vertices and 924 edges in this graph

\section{Large Straight-Line Drawings}

Traditional aesthetics for straight-line drawings of general undirected graphs, such as display symmetry, avoiding edge crossings, and uniform edge lengths [4], are in strong conflict for large graphs. Fortunately, this is relatively unimportant, since actual edge connections are perceptible only on a local scale. On the whole, the location of vertices gains more significance as it becomes the dominant factor for judging graph-theoretic distances. Therefore, if a proper overview of a graph is to be achieved these distances should be approximated by Euclidean distances between vertices in a drawing.

A function, that for a given pair of vertices returns the length of the shortest path between them, satisfies metric properties:

- $f(a, b)=f(b, a)$, true for an undirected graph

$-f(a, b) \geq 0$, with $f(a, b)=0$, if and only if $a=b$

$-f(a, b) \leq f(a, c)+f(c, b)$, for all vertices $a, b, c$. If $c$ is a vertex on the shortest path between $a$ and $b$ then $f(a, b)=f(a, c)+f(c, b)$. Otherwise, $f(a, b)>$ $f(a, c)+f(c, b)$ cannot hold because a path through $c$ would be shorter.

These properties are preserved for a disconnected graph if the length of the shortest path between vertices from separate components is considered to be infinite (although, for practical purposes a value greater than the longest path in all components will suffice). This function is a dissimilarity metric for graphs.

Multidimensional Scaling (MDS) is a standard multivariate analysis method [9], that can be applied to a given dissimilarity metric and a data collection, to yield a matching configuration of points, with each point representing a single 
data object. Least-squares metric MDS uses a numerical minimisation process to ensure that Euclidean distances in the configuration approximate dissimilarities between corresponding data objects. For the graph metric this will give the desired result of approximating graph-theoretic distances. This approach to graph drawing was first adopted by Kruskal and Seery [17].

Incremental MDS [3] is a least-squares metric MDS algorithm that is capable of laying out even very large data collections (100,000 objects) in a satisfactory time on a standard workstation. This performance is achieved at the slight expense of layout quality - details of this mechanism can be found in the original paper. For smaller data collections (up to thousands of objects) it is feasible to resort to the standard, non-incremental mode. At this level the algorithm is essentially equivalent to that of Kamada and Kawai [15]. It emulates a fully connected spring system with one anchor point for every vertex. The relaxed length of a spring connecting two points is taken to be the dissimilarity between the corresponding pair of vertices (shortest path length). The actual length of the spring is the Euclidean distance between its two anchor points. The algorithm attempts to arrive at a minimum energy state (an optimal configuration) by reducing the disparity between actual and desired lengths. This process is iterative and terminates when the amount of improvement becomes negligible.

The energy potential of the spring system is given by a loss function

$$
E=\sum_{r<s} \frac{\left(d_{r s}-\hat{d}_{r s}\right)^{2}}{\hat{d}_{r s}^{2}}
$$

that is being minimised. In this formula $d_{r s}$ denotes the actual length of the spring connecting points $r$ and $s, \hat{d}_{r s}$ is the relaxed length of the spring. Since contributions of individual springs are normalised, any deviation in distance between adjacent vertices is penalised more than the same error for non-adjacent vertices. Therefore, a straight-line drawing based on an optimal vertex layout will have uniform edge lengths. At the same time an even spread of vertices is achieved, because distances between non-adjacent vertices tend to be proportional to their shortest path lengths. These properties are illustrated by a graph drawing in Figure 3. This algorithm does not explicitly minimise edge crossings or maximise drawing symmetry, however one or both of these aesthetics might be correlated with the optimisation criterion of preserving graph-theoretic distances, for certain graphs. An optimal layout of a 20x20 grid in Figure 2 serves as an example.

MDS can be used to embed a graph in an Euclidean space of any dimension. $2 \mathrm{D}$ layouts are an obvious choice, however 3D drawings can be advantageous, particularly for strongly connected graphs: in three dimensions edge crossings are less likely to occur. Also, graphs with a large number of disconnected components can benefit from a three dimensional representation. Please note how small components are arranged on a hemisphere around the main component in Figure 4. This arrangement will be squashed to a semicircle in two dimensions.

For converting an MDS layout to a 2D drawing we have created a simple viewer. It has facilities for labelling vertices, colouring vertices and edges, and 
storing the drawing as an image file. Figures 1 and 3 were created with this program. Interactive examination is crucial for gaining an understanding of an abstract 3D representation. A combination of a 3D scene description language and a viewer achieves this goal in a flexible way. We have decided to use Virtual Reality Modelling Language (VRML) [6], which can be viewed in a web browser with an appropriate plug-in or a stand-alone VRML viewer. This results in a very portable method of disseminating these drawings. Figures 4-7 are snapshots taken along a sample navigation path.

Andalman et al have explored the use of MDS for creating VRML visualisations of synthetic image collections - Design Galleries [1]. A dissimilarity metric capturing perceptual distance between images has been used. An image collection can be seen to be equivalent to a complete weighted graph, with images as vertices, and dissimilarities between pairs of images as edge weights. A visualisation consists of images arranged at the coordinates calculated by MDS. To avoid clutter, edges are omitted as their weights are approximated by image distances.

\section{Evaluation}

It is customary for protein interaction graphs to be drawn manually or presented in a tabular form. This approach is not suitable for large data sets, however. To our knowledge, it is the first time an automatic graph drawing technique has been applied in this context. The visualisation of all of the proteins and interactions between them has provided us with a new means in which to study the data stored.

Upon inspection of the clusters in the VRML representation of yeast interactions (Figure 4 represents a starting point of exploration), it became apparent that there are several errors in the database. These errors were visible to a 'yeast' domain expert who recognised that some patterns were incorrect. In the first instance, the protein ALG5 is shown in Figure 5 to interact with many proteins,

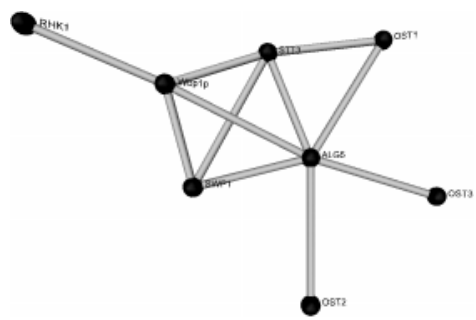

Fig. 5. Neighbourhood of ALG5 protein

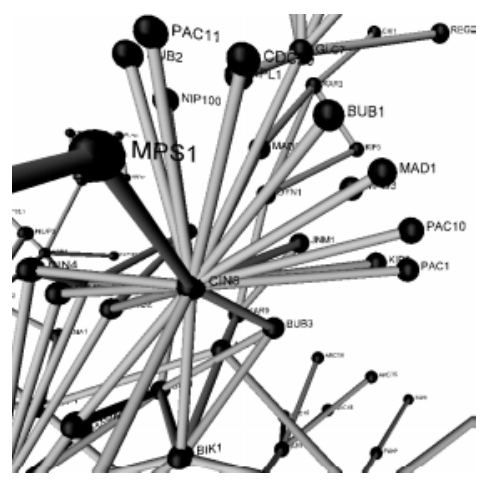

Fig. 6. Neighbourhood of CIN8 protein 


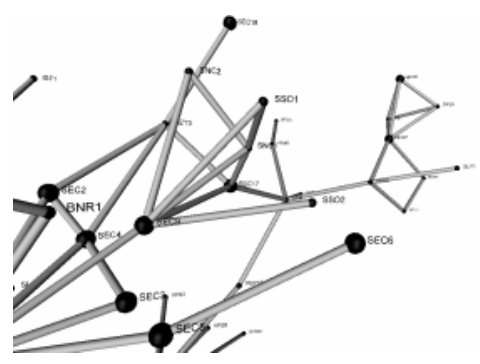

Fig. 7. Neighbourhood of SEC proteins. Spheres representing proteins of interest have larger diameter

when in fact it interacts with only one of the displayed proteins, WBP1. In the second instance, it was observed that the protein CIN8 exhibited far more interactions than actually exist, as seen in Figure 6. Both of these errors were undetected until the interactions were visualised. They have been attributed to experiments relying on genetic methods, which can sometimes produce false positive results.

The location of proteins within the graph drawing appears to be logical. For example, the SEC proteins, responsible for vesicle mediated transport, are located together in Figure 7; proteins involved in signal transduction are also grouped. The aspect of the layout that concerned the biological end user, was the question of whether vertex proximity in the drawing equated to close cellular location of the corresponding proteins. This is not true for cases where the proteins in question do not directly interact; however such proteins are unlikely to be close in the drawing anyway, as they are not adjacent in the graph. Another related aspect, that was pleasing to the user, was the scarcity of overly long links between pairs of proteins, that cross over many other links.

When analysing the protein interaction graphs, the end users found it most productive to view both the $2 \mathrm{D}$ and $3 \mathrm{D}$ drawings synchronously. It is possible to lose context and become 'lost in space' while navigating a 3D representation, so the $2 \mathrm{D}$ diagram is useful for regaining orientation. Using this approach it is then possible to study the more complex regions of nodes with many spokes like SNP1, and very highly connected complexes such as the NUP complex.

The data represented is highly connected, and the complexity can make certain regions difficult to comprehend. Filters were used to restrict the amount of data displayed. This came in the form of how believable the interaction actually is. Each interaction was assigned a number depending on the confidence in the experimentation. A series of graphs were then created from the skeleton of definite interactions, with the layers of less certain interactions added in different colours. This technique allowed the biologists to view the data in a simple form and then increase the complexity to add to their understanding, while preserving their mental map between stages [10].

Another application of graph drawing has been the representation of comparative studies between organisms. The $2 \mathrm{D}$ drawing in Figure 1 has been colour 
coded to display a comparison between the Eukaryotes: yeast and C. elegans. The proteins responsible for making yeast a single cellular organism are obviously not present in C. elegans (a multi-cellular nematode worm), so are coloured differently. This diagram highlights the protein interactions of the kinetochore complex, which has a mitotic function in yeast. This representation has allowed the rapid identification of pathways and complexes unique to yeast, and also conserved eukaryotic interactions. Comparative analysis is useful as it provides a mechanism to pinpoint the function of proteins. (Eukaryotes are organisms with complex compartmentalised cells, ranging from single cell lower eukaryotes such as yeast to multicellular higher eukaryotes which are anything more complex than sponges. The other two domains of life are Eubacteria and Archaea, which both encompass single-celled life.)

\section{$5 \quad$ Further Work}

3D graph drawings in VRML are very interactive, and encourage exploration of the underlying data. On the other hand, static 2D drawings that we have produced so far are of limited usefulness. They serve as an overview of the graph structure, but fail to convey detailed relationships. To alleviate this situation a zoom facility is needed. Fisheye magnification seems most appropriate to use, as it preserves context. The SHriMP layout adjustment algorithm [19] avoids excessive distortion to peripheral objects, and we shall adopt it in the future.

The advantage of using MDS for calculating layouts of protein interactions is that it is not specific to a graph representation of these data. Instead of a dissimilarity metric based on graph-theoretic distances, we can use one that takes the location of proteins within the cell into account. A comparison of layouts resulting from both data representations might help to clarify the relationship between the location of a protein and its interactions.

\section{Conclusions}

A combination of MDS and a graph-theoretic metric appears to constitute an ideal graph drawing technique for protein interaction graphs, and others of similarly high level of complexity. The drawings produced exhibit aesthetics typical to that of spring embedder algorithms, and additionally preserve the underlying graph topology.

Automatically generated 2D and 3D graph drawings are a welcome alternative to existing methods of analysing protein interactions. They impose a tangible representation, to which users can readily relate, upon these complex data. These visualisations are invaluable for discovering data inconsistencies, and carrying out comparative studies. 


\section{Acknowledgements}

Wojciech Basalaj's work is supported by Trinity College, Cambridge, and by the Overseas Research Students Awards Scheme. Karen Eilbeck's work is supported by a BBSRC case studentship with GlaxoWellcome. Thanks to Dr Ian Dix, for his yeast protein domain expertise.

\section{References}

[1] B. Andalman et al. Design gallery browsers based on 2D and 3D graph drawing (demo). In Proc. of Graph Drawing '97, volume LNCS 1353, pages 322-329, September 1997.

[2] P. L. Bartels et al. A protein linkage map of Escherichia coli bacteriophage -T7. Nature Genetics, 12:72-77, 1996.

[3] W. Basalaj. Incremental multidimensional scaling method for database visualization. In Proc. of Visual Data Exploration and Analysis VI, volume SPIE 3643, pages 149-158, January 1999.

[4] G. Di Battista et al. Algorithms for drawing graphs: an annotated bibliography. Computational Geometry: Theory and Applications, 4:235-282, 1994.

[5] F. R. Blattner et al. The complete genome sequence of Escherichia coli K-12. Science, 277:1453-74, 1997.

[6] R. Carey and G. Bell. The Annotated VRML 2.0 Reference Manual. AddisonWesley Developers Press, Reading, MA, USA, 1997.

[7] R. A. Clayton et al. The first genome from the third domain of life. Nature, 387:459-462, 1997.

[8] F. S. Collins et al. New goals for the U.S. Human Genome Project: 1998-2003. Science, 282:682-9, 1998.

[9] T. F. Cox and M. A. A. Cox. Multidimensional Scaling. Chapman \& Hall, London, 1994.

[10] P. Eades et al. Preserving the mental map of a diagram. In COMPUGRAPHICS '91, pages 34-43, September 1991.

[11] K. Eilbeck et al. INTERACT: An object oriented protein-protein interaction database. In Proc. of ISMB (in press), 1999.

[12] The C. elegans Sequencing Consortium. Genome sequence of the nematode C. elegans: a platform for investigating biology. Science, 282:2012-8, 1998.

[13] M. Fromont-Racine, J.C. Rain, and P. Legrain. Toward a functional analysis of the yeast genome through exhaustive two-hybrid screens. Nature Genetics, 16:277-82, 1997.

[14] A. Goffeau et al. Life with 6000 genes. Science, 274:563-7, 1996.

[15] T. Kamada and S. Kawai. An algorithm for drawing general undirected graphs. Information Processing Letters, 31:7-15, 1989.

[16] P. D. Karp et al. Ecocyc: Encyclopedia of Escherichia coli genes and metabolism. Nucleic Acids Res, 27:55, 1999.

[17] J. B. Kruskal and J. B. Seery. Designing network diagrams. In Proc. of the First General Conference on Social Graphics, pages 22-50, October 1978.

[18] H. W. Mewes et al. MIPS: a database for genomes and protein sequences. Nucleic Acids Res, 27:44-8, 1999.

[19] M.-A. D. Storey and H. Mueller. Graph layout adjustment strategies. In Proc. of Graph Drawing '95, volume LNCS 1027, pages 487-499, September 1995. 